\title{
openheart Anomalous coronary artery origin from the opposite sinus in patients with bicuspid aortic valve: comparison with tricuspid aortic valve
}

\author{
Saarwaani Vallabhajosyula, ${ }^{1}$ Margaret Fuchs, ${ }^{2}$ Li-Tan Yang, ${ }^{3}$ Jose Medina Inojosa, ${ }^{3}$ \\ Tanya H Tajouri, ${ }^{4}$ Maurice Enriquez-Sarano, ${ }^{3}$ Sabrina D Phillips, ${ }^{1}$ Rajiv Gulati, ${ }^{3}$ \\ Kyle W Klarich, ${ }^{1}$ Hector Michelena (D) ${ }^{3}$
}

To cite: Vallabhajosyula S, Fuchs M, Yang L-T, et al. Anomalous coronary artery origin from the opposite sinus in patients with bicuspid aortic valve: comparison with tricuspid aortic valve. Open Heart 2021;8:e001567. doi:10.1136/ openhrt-2020-001567

SV and MF contributed equally.

Received 29 December 2020 Accepted 25 May 2021

\section{Check for updates}

C) Author(s) (or their employer(s)) 2021. Re-use permitted under CC BY-NC. No commercial re-use. See rights and permissions. Published by BMJ.

${ }^{1}$ Department of Cardiovascular Diseases, Mayo Clinic, Rochester, Minnesota, USA ${ }^{2}$ Department of Cardiovascular Medicine, Cleveland Clinic, Cleveland, Ohio, USA

${ }^{3}$ Department of Cardiovascular Medicine, Mayo Clinic,

Rochester, Minnesota, USA

${ }^{4}$ Department of Cardiology, Sentara Healthcare Inc, Harrisonburg, Virginia, USA

Correspondence to

Dr Hector Michelena; michelena. hector@mayo.edu

\section{ABSTRACT}

Objective To compare the prevalence and patterns of anomalous coronary artery origin from the opposite sinus (ACAOS) in patients with bicuspid aortic valve (BAV) and tricuspid aortic valve (TAV).

Methods Retrospective review of consecutive patients with surgically excised BAV and TAV was performed from 1994 to 2015. Clinical notes, echocardiograms, coronary angiograms, CT angiographies, and pathology reports were reviewed. ACAOS included right coronary artery from the left cusp, left circumflex artery from the right cusp and left main or left anterior descending artery from the right cusp.

Results 2371 (years 1994-2015) and 1679 (years 20092015) consecutive patients with pathology-confirmed BAV and TAV, respectively, and defined preoperative coronary anatomy were identified. A left dominant coronary circulation was present in $386(18 \%)$ patients with BAV and $179(11 \%)$ patients with TAV $(p<0.001)$. ACAOS was identified in $43(1.8 \%)$ patients with BAV and $15(0.9 \%)$ patients with TAV, $p=0.02$. Among patients with BAV and ACAOS, the most common phenotype was right-left fusion ( $n=34,79 \%)$ with present raphe $(n=36,84 \%)$, with no association between BAV phenotype and ACAOS type. On multivariate analysis, BAV status and size of the mid-ascending aorta were independently associated with ACAOS (OR 3.29; Cl 1.26 to 8.6; $p=0.02$; OR 0.93; $\mathrm{Cl} 0.87$ to $0.98 ; p=0.01$; respectively). Only two patients with ACAOS, one with BAV and one with TAV, had a perioperative coronary ischaemic event.

Conclusions The prevalence of the potentially malignant ACAOS is significantly higher (threefold higher odds) in patients with BAV as compared with TAV, yet remains uncommon in absolute terms. Most patients with BAV and ACAOS had right-left cusp fusion and present raphe. Perioperative coronary events are rare in patients with ACAOS.

\section{INTRODUCTION}

Coronary artery anomaly (CAA) is an uncommon entity with abnormal origin, course or calibre of a coronary artery. The overall prevalence of CAAs cited in the

\section{Key questions}

What is already known about this subject?

- Individuals with a bicuspid aortic valve (BAV) have long been noted to have clinically benign variations in coronary anatomy compared with the general population, including a higher prevalence of left coronary artery-dominant circulation, short left main coronary artery and separate ostia of the left anterior descending and circumflex coronary arteries. However, whether the prevalence of clinically significant coronary artery anomalies such as anomalous origin of a coronary artery from the opposite sinus (ACAOS) is higher in patients with BAV remains controversial.

What does this study add?

- The current study is the largest evaluating coronary anomalies in BAV and shows a clinically and statistically significant higher prevalence of ACAOS (two times more prevalent, approximately $2 \%$ ) in patients with BAV versus their tricuspid counterparts. All varieties of ACAOS are seen in patients with BAV and the presence of ACAOS is related, although uncommonly, to perioperative complications.

How might this impact on clinical practice?

- The current study advocates for coronary anatomy characterisation before cardiac surgery in patients with BAV regardless of age and alerts clinicians following patients with BAV of their higher prevalence of clinically significant coronary anomalies, such as ACAOS.

literature varies significantly, from $0.2 \%$ to $5.8 \% .^{1-3}$ Differences in diagnostic imaging modalities and definitions of CAA likely account for much of this variation. Several CAA classification schemes have been devised, some based on anatomical features and others on perceived risk of adverse event. ${ }^{24}$ While a few variations are recognised as clinically deleterious (eg, anomalous coronary artery from the pulmonary artery), it is 
important to clarify patterns that are considered 'benign variations of normal' and those that are truly 'anomalous'. ${ }^{4}$ Variation in the length of the left main coronary artery (LMCA), including the extreme of absent LMCA with separate ostia of the left anterior descending (LAD) and left circumflex arteries (LCx), has a benign clinical course and is considered a normal variant. ${ }^{45}$ High coronary take-off is another CAA of uncertain clinical significance; while isolated case reports have described sudden cardiac death potentially attributable to high take-off, many experts consider this anatomical feature a variation of little clinical significance..$^{5-7}$ In comparison, anomalous origination of a coronary artery from the opposite sinus (ACAOS) is uncommonly observed in the general population and has been recognised for its potential to cause symptoms of cardiac ischaemia and rarely, sudden cardiac death. ${ }^{4}$ Because of its 'malignant' potential, ACAOS should be classified as a true CAA. ${ }^{458}$

Individuals with a bicuspid aortic valve (BAV) have long been noted to have variations in coronary anatomy compared with the general population. It is well established that patients with BAV have a higher prevalence of left dominant coronary circulation, approximately $25 \%$ compared with an estimated $10 \%$ in normal controls. ${ }^{910}$ Variants of normal coronary anatomy have also recently been reported to have higher prevalence in the BAV population, including significantly shorter LMCA, higher prevalence of absent LMCA and a correlation between absent LMCA and BAV with right-left cusp fusion and absent raphe. ${ }^{11} 12$ The prevalence of ACAOS in patients with BAV is less certain. ${ }^{12}{ }^{13}$ Given the clinical importance of ACAOS and its uncertain prevalence in the BAV population, we studied a large cohort of patients with BAV and tricuspid aortic valve (TAV) with defined coronary anatomy in order to describe the prevalence of ACAOS and its association with BAV phenotype.

\section{METHODS}

\section{Patient selection, consent and data collection}

A retrospective review was performed of consecutive patients with BAV undergoing aortic valve replacement (AVR) from January 1994 to November 2015 at our institution. Patients were identified based on pathology reports describing the explanted aortic valve as bicuspid. A control group of consecutive patients with pathologyconfirmed surgically excised TAV from January 2009 to December 2015 was also identified. Medical records were reviewed, including clinical notes, echocardiograms, cardiac catheterisation, coronary computed tomographic angiogram (CTA), surgical notes and pathology reports. Demographic, clinical and haemodynamic data at the time of presurgical evaluation were collected categorically for statistical analysis.

\section{Preoperative imaging studies}

Preoperative coronary angiogram or CTA reports were reviewed for all patients undergoing surgical AVR. The

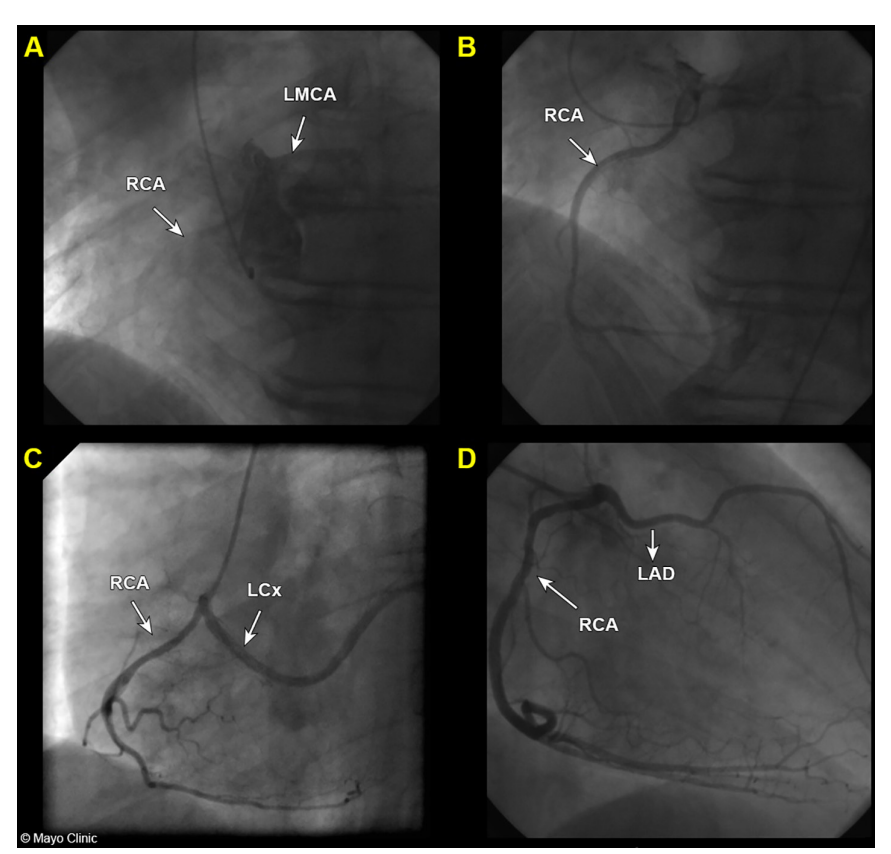

Figure 1 Patterns of ACAOS. Panel (A) and (B): origin of right RCA from left cusp; panel (C): origin of LCx from right cusp; panel (D): origin of LAD from right cusp. ACAOS, anomalous origination of a coronary artery from the opposite sinus; LAD, left anterior descending artery; LCx, left circumflex artery; LMCA, left main coronary artery; RCA, right coronary artery.

pattern of coronary artery dominance was noted in each study; coronary artery dominance was described as right dominant when the posterior descending artery (PDA) originated from the right coronary artery (RCA), and left dominant when the PDA originated from the LCx. In patients with balanced circulation, the PDA originated from the RCA, and a large posterolateral branch from the LCx reached to or near the posterior interventricular groove. The presence or absence of CAA was also based on the imaging report, the patient's diagnosis code and/ or electronic keyword search of the patient's chart. When a patient was identified as having a CAA, the angiographic or radiological images were reviewed de novo by an experienced observer to confirm the diagnosis (RG). Patients were considered to have ACAOS if one of three patterns were observed: (1) origin of the RCA from the left cusp (figure 1A,B), (2) origin of the LCx from the right cusp (figure 1C and figure 2) and(3) origin of the LMCA or LAD (figure 1D) from the right cusp.

Preoperative echocardiogram reports were reviewed for all patients undergoing AVR. When there was coincident BAV and CAA, preoperative echocardiographic images were reviewed de novo by an experienced observer to establish the BAV phenotype (MMF, KWK and HIM). Aortic dimensions were measured de novo leading edge to leading edge at end-diastole for these patients. ${ }^{14}$ The presence or absence of BAV raphe was determined from pathological specimen reports. Surgical complications in ACAOS were assessed by reviewing postoperative hospital records and dismissal summary. 


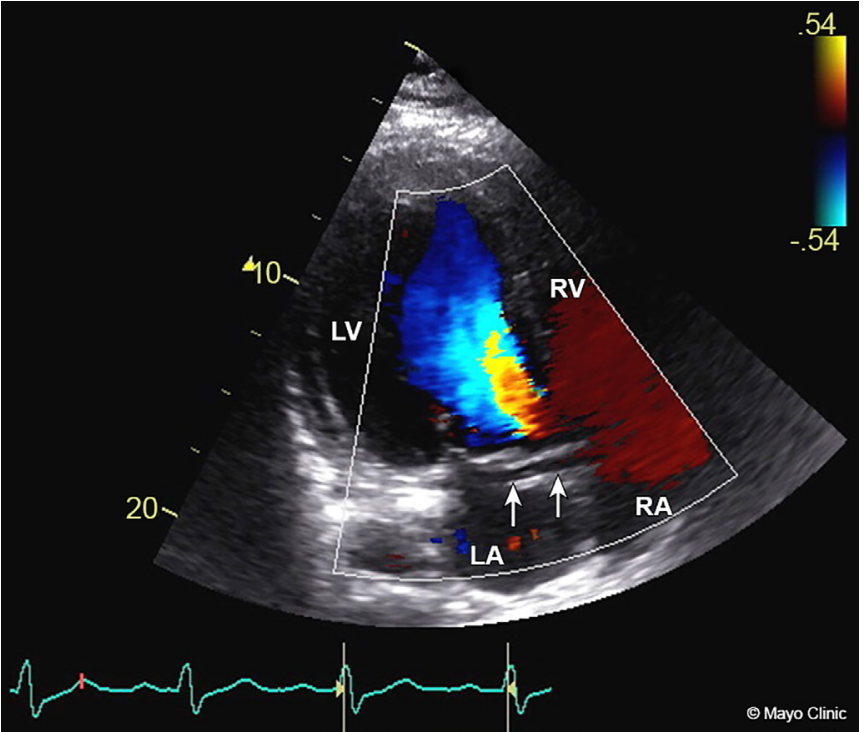

Figure 2 Systolic still-frame of apical four-chamber view with an anterior tilt demonstrating the anomalous left circumflex artery (arrows) originating from the right sinus in this study patient. LA, left atrium; LV, left ventricle; RA, right atrium; $\mathrm{RV}$, right ventricle.

\section{Outcomes of interest}

Primary outcomes of interest included prevalence and distribution of patterns of ACAOS in patients with pathology-confirmed BAV versus TAV. Other outcomes of interest included clinical predictors of ACAOS, its association with BAV phenotype and analyse perioperative risk of ACAOS in patients with BAV and patients with TAV undergoing AVR.

\section{Patient and public involvement}

Patients and/or public were not involved in the design, or conduct, or reporting, or dissemination plans of our research.

\section{Statistical analysis}

Categorical variables were reported as percentages, and continuous variables were reported as mean $\pm \mathrm{SD}$ or median (IQR) for skewed data. Categorical variables were compared using the Fisher exact test, and continuous variables were compared with a two-sided, unpaired $t$ test. First, the correlation between sinus of Valsalva and midascending aorta measurements was assessed given that collinearity was hypothesised, ${ }^{15}$ and this was not found to be significant $(p=0.38)$. Then, independent predictors of ACAOS were analysed using logistic regression modelling in the entire cohort of BAV and TAV with preoperative imaging. OR was reported for each univariate determinant. Stepwise multivariable analysis was performed including variables when $\mathrm{p}<0.1$. All statistical analyses were performed using commercially available software (JMP V.14.1.0, SAS Institute, Cary, North Carolina, USA; and GraphPad V.7.04, GraphPad Software, San Diego, California, USA). For all statistical analyses, $\mathrm{p}<0.05$ was considered statistically significant.

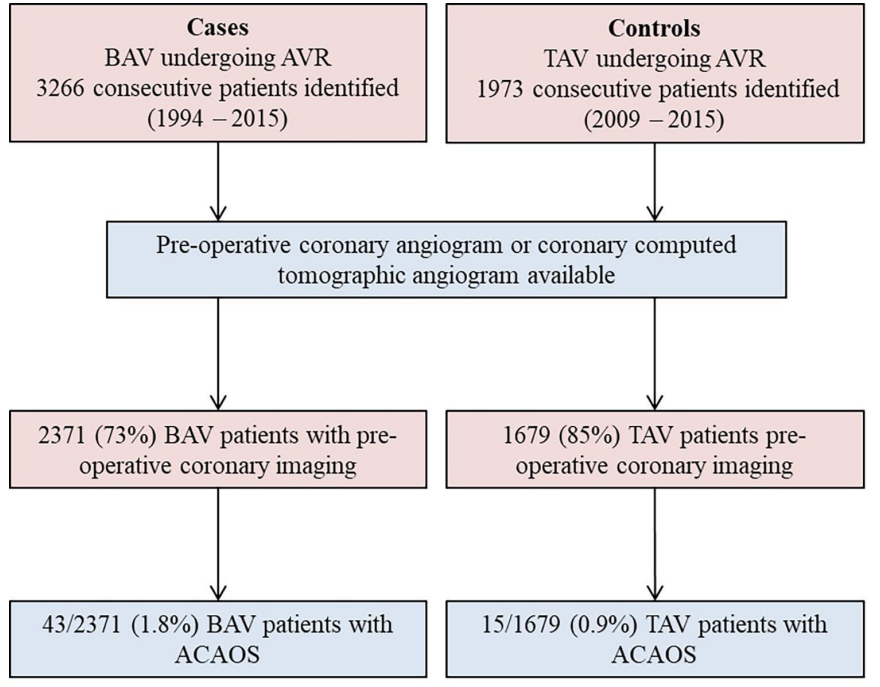

Figure 3 Study flow. ACAOS, anomalous coronary artery from opposite sinus; AVR, aortic value replacement; BAV, bicuspid aortic value; TAV, tricuspid aortic valve.

\section{RESULTS}

There were 3266 consecutive patients with surgically excised BAV and 1973 consecutive patients with surgically excised TAV. Preoperative coronary angiograms or coronary CTAs were available for 2371 (73\%) patients with BAV and $1679(85 \%)$ patients with TAV, and only these patients were studied further (figure 3). Within these patients, echocardiogram reports were available in $1788(75 \%)$ patients with BAV and 1662 (99\%) patients with TAV. The demographics of patients with and without coronary imaging are provided in table 1. As expected, both patients with BAV and patients with TAV with coronary imaging were older, had more risk factors and known coronary disease than patients without coronary imaging (table 1).

\section{Baseline demographics}

Table 2 provides baseline and echocardiographic demographics of 2371 patients with BAV and 1679 patients with TAV. Average age at AVR was $63 \pm 13$ years for BAV, compared with $77 \pm 8$ for TAV, $\mathrm{p}<0.001$. Male sex accounted for $74 \%$ and $63 \%$ of patients undergoing BAV and TAV replacement, respectively, $\mathrm{p}<0.001$. BAV had an average sinus of Valsalva and mid-ascending aortic dimension of $39 \pm 6$ and $42 \pm 7 \mathrm{~mm}$, respectively, compared with $35 \pm 4$ and $36 \pm 4 \mathrm{~mm}$ in the TAV group, both $\mathrm{p}<0.001$. Indication for BAV-AVR was aortic stenosis (AS) in $52 \%$, aortic regurgitation (AR) in $11 \%$ and mixed AS/AR in $36 \%$. In contrast, TAV-AVR was performed predominantly due to AS in $94 \%$ patients with a smaller proportion of AR or mixed AS/AR, all $\mathrm{p}<0.001$ as compared with BAV. Concomitant ascending aortic replacement was performed in $26 \%$ of BAV vs $1 \%$ of TAV, $\mathrm{p}<0.001$.

\section{Coronary anatomy}

Among BAV, 386 (18\%) had a left dominant coronary artery circulation, compared with 179 (11\%) patients in 


\begin{tabular}{|c|c|c|c|c|}
\hline \multicolumn{2}{|l|}{ Characteristic } & $\begin{array}{l}\text { Coronary imaging } \\
\text { present }\end{array}$ & $\begin{array}{l}\text { Coronary imaging } \\
\text { absent }\end{array}$ & $p$ value \\
\hline \multicolumn{2}{|c|}{ (A) Patients with BAV grouped by coronary imaging } & $\mathrm{n}=2371$ & $\mathrm{n}=895$ & $<0.001$ \\
\hline \multicolumn{2}{|l|}{ Age at valve replacement, years } & $63 \pm 13$ & $50 \pm 19$ & $<0.001$ \\
\hline \multicolumn{2}{|l|}{ Sex, male } & $1752(74)$ & $645(72)$ & 0.27 \\
\hline \multirow[t]{5}{*}{ Medical history } & Hypertension & $1388(59)$ & $361(40)$ & $<0.001$ \\
\hline & Hyperlipidaemia & $1198(51)$ & $278(31)$ & $<0.001$ \\
\hline & Diabetes mellitus & $370(16)$ & $95(11)$ & $<0.001$ \\
\hline & Coronary artery disease & $1553(66)$ & $249(28)$ & $<0.001$ \\
\hline & Chronic kidney disease & $178(8)$ & $56(6)$ & 0.2 \\
\hline \multirow[t]{3}{*}{ Surgical procedure performed } & AVR alone & $1763(5)$ & $594(66)$ & $<0.001$ \\
\hline & $\begin{array}{l}\text { AVR with concomitant root repair/ } \\
\text { replacement }\end{array}$ & $605(26)$ & $301(34)$ & \\
\hline & Heart transplant & $2(0.1)$ & $1(0.1)$ & \\
\hline \multicolumn{2}{|c|}{ B. Patients with TAV grouped by coronary imaging } & $n=1679$ & $\mathrm{n}=294$ & $<0.001$ \\
\hline \multicolumn{2}{|l|}{ Age at valve replacement, years } & $77 \pm 8$ & $75 \pm 9$ & 0.002 \\
\hline \multicolumn{2}{|l|}{ Sex, male } & $1063(63)$ & $186(63)$ & 0.93 \\
\hline \multirow[t]{5}{*}{ Medical history } & Hypertension & $1480(88)$ & $243(83)$ & 0.01 \\
\hline & Hyperlipidaemia & 1409 (84) & $233(79)$ & 0.04 \\
\hline & Diabetes mellitus & $623(37)$ & $115(39)$ & 0.52 \\
\hline & Coronary artery disease & 1594 (95) & $227(77)$ & $<0.001$ \\
\hline & Chronic kidney disease & $440(26)$ & $87(30)$ & 0.23 \\
\hline \multirow[t]{3}{*}{ Surgical procedure performed } & AVR alone & 1657 (99) & $290(98)$ & $<0.001$ \\
\hline & $\begin{array}{l}\text { AVR with concomitant root repair/ } \\
\text { replacement }\end{array}$ & $22(1)$ & $5(2)$ & \\
\hline & Heart transplant & $0(0)$ & $0(0)$ & \\
\hline
\end{tabular}

Data expressed as mean \pm SD or as number (percentage).

AVR, aortic valve replacement; BAV, bicuspid aortic valve; TAV, tricuspid aortic valve.

the TAV group $(\mathrm{p}<0.001$, table 2$)$. ACAOS was detected in $43(1.8 \%)$ of BAV and $15(0.9 \%)$ of TAV $(\mathrm{p}=0.02$, figure 4, table 3 ). The most common ACAOS in the BAVACAOS group was anomalous LCx from the right cusp $(n=21,49 \%)$. Anomalous RCA from the left cusp was present in $14(33 \%)$ patients, and anomalous LMCA or LAD from the right cusp was present in $8(19 \%)$. In the TAV group, LCx from the right cusp occurred in 8 (53\%) patients, anomalous RCA from the left cusp occurred in $4(27 \%)$ patients, and LMCA or LAD from the right cusp in $3(20 \%)$ (table 3$)$. There was no statistically significant difference in pattern of ACAOS between the two groups.

\section{Surgical procedure and associated features}

The most common surgical indication for patients with BAV-ACAOS was AS $(n=27,62 \%)$, followed by mixed AS/AR ( $=9,21 \%)$, AR $(n=5,12 \%)$ and infective endocarditis $(n=2,5 \%)$. Ascending aorta replacement was required in $14(33 \%)$ (table 3). Patients with TAV-ACAOS had a surgical indication of AS in $11(73 \%)$ and AS/ $\mathrm{AR}$ in $4(27 \%)$. None of the patients with TAV-ACAOS required ascending aorta replacement. Two patients had a coronary-related perioperative complication: (1) one patient with BAV and anomalous RCA from the left sinus underwent aortic valve and root replacement; right ventricular dysfunction was noted by intraoperative transoesophageal echocardiogram at the time of chest closure, felt to be related to compression of the anomalous RCA, requiring bypass graft to the RCA; and (2) one patient with TAV and anomalous LMCA from the right cusp underwent AVR with attempted coronary unroofing; the patient developed an abrupt drop in left ventricular ejection fraction, prompting coronary artery bypass grafting to the LAD and LCx. Both patients with perioperative complications had otherwise unremarkable hospital courses and survived to hospital dismissal. Coincident congenital heart disease was present in four patients with BAV-ACAOS (table 3). Pathology findings consistent with disease of the aortic media were identified in six patients with BAV. Two patients with TAV had other pathological diagnoses (amyloidosis and hypertrophic cardiomyopathy). 


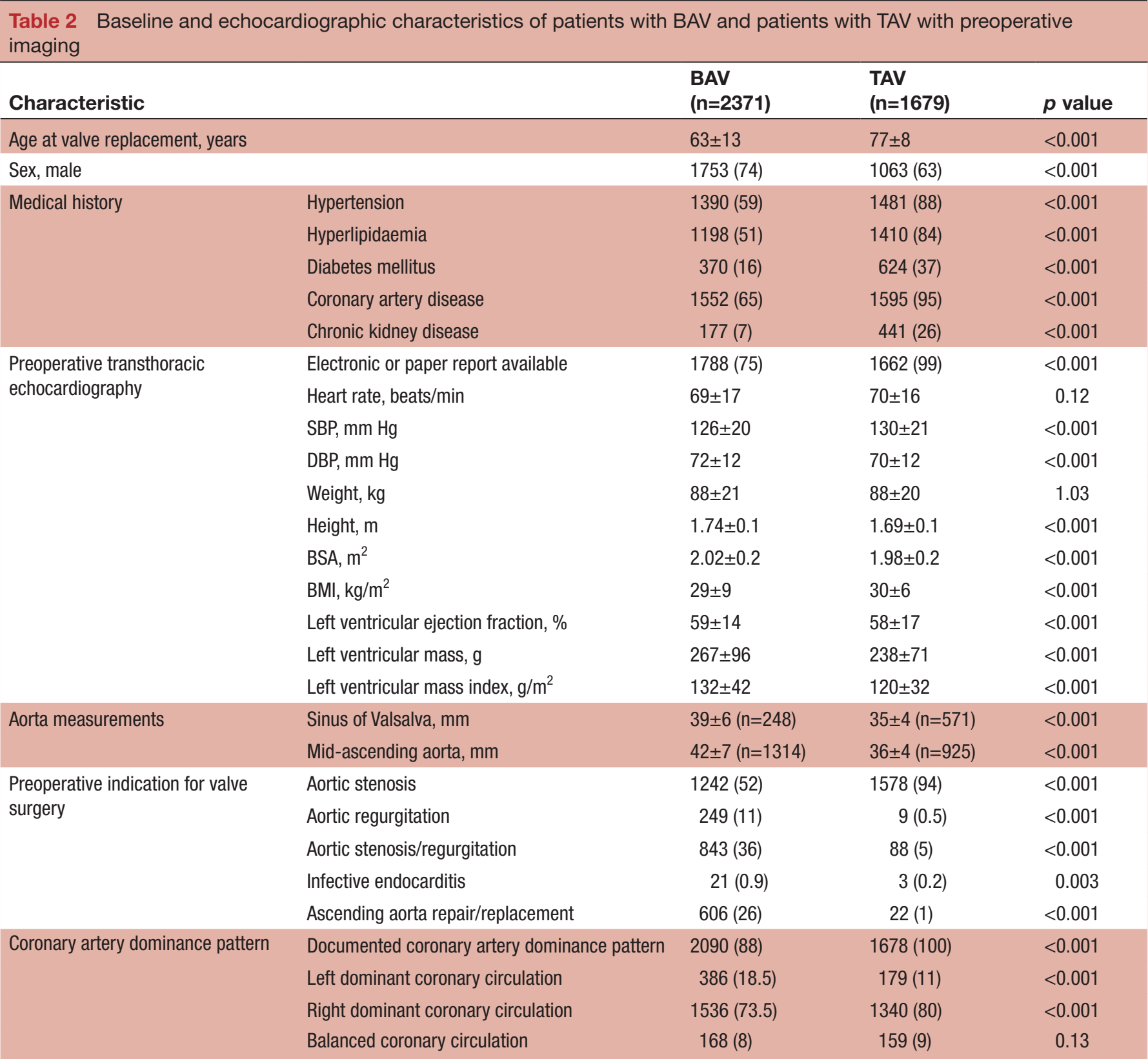

Data expressed as mean \pm SD or as number (percentage).

BAV, bicuspid aortic valve; BMI, body mass index; BSA, body surface area; DBP, diastolic blood pressure; SBP, systolic blood pressure; TAV, tricuspid aortic valve.

\section{Coincident BAV-ACAOS}

In BAV-ACAOS, right-left cusp fusion was observed in 34 (79\%) patients, while right-non cusp fusion was present in $8(19 \%)$ patients and left non-cusp fusion in $1(2 \%)$. Excised BAVs were observed to have raphe in $36(84 \%)$ specimens, while $4(9 \%)$ specimens had no raphe. Three pathology reports did not delineate presence or absence of raphe. All patterns of ACAOS were represented in patients with all BAV phenotypes, and there was no association observed. In the logistic regression model, younger age, BAV status and smaller mid-ascending aorta measurements were univariately associated with presence of ACAOS ( $\mathrm{p}=0.001,0.02$ and 0.08 , respectively), whereas male sex, sinus of Valsalva measurements, AS or regurgitation, left dominant coronary circulation and concomitant root repair/replacement were not (table 4). In multivariate analysis, BAV status and size of mid-ascending aorta were independently associated with ACAOS (OR 3.29; CI 1.26 to 8.6; $\mathrm{p}=0.02$ and OR 0.93; CI 0.87 to $0.98 ; \mathrm{p}=0.01$, respectively).

\section{DISCUSSION}

This study describes the prevalence and patterns of ACAOS in the largest reported cohort of patients with pathology-confirmed BAV and CAA. The principal findings are: (1) as compared with TAV, ACAOS was two times more prevalent in the BAV population (threefold higher 


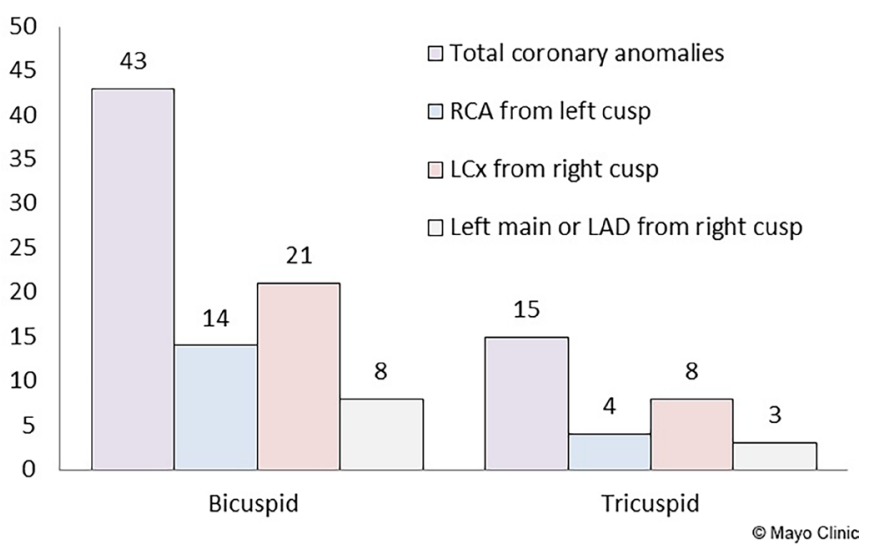

Figure 4 Distribution of ACAOS in patients with BAV and patients with TAV. ACAOS, anomalous origination of a coronary artery from the opposite sinus; BAV, bicuspid aortic valve; LAD, left anterior descending artery; $L C x$, left circumflex artery; RCA, right coronary artery; TAV, tricuspid aortic valve. odds); (2) all patterns of ACAOS were observed in BAV, most commonly anomalous LCx from the right cusp (also most common in TAV), and their most common phenotype was right-left fusion with present raphe and (3) only $3.4 \%$ patients with ACAOS (1 BAV, $1 \mathrm{TAV}$ ) had an adverse perioperative coronary event.

\section{Development of coronary anatomy}

The developmental relationship between BAV and CAA is not well understood. The aortic valve develops from endocardial tissue that first septates the aortic trunk into pulmonic and systemic outlets before forming the arterial valves. ${ }^{16} \mathrm{BAV}$ results from either fusion of adjacent cusps or failure of cusp division, ${ }^{17}$ and it is possible that the two most common phenotypes (right-left and right-non) represent different aetiological entities that are genotypically distinct. ${ }^{18}$ While previously the coronary arteries were thought to develop via angiogenesis (ie, growing out from the aortic root), more recent studies support

\begin{tabular}{|c|c|c|c|c|}
\hline Characteristic & & $\begin{array}{l}\text { BAV } \\
\mathrm{n}=43\end{array}$ & $\begin{array}{l}\text { TAV } \\
\mathrm{n}=15\end{array}$ & $p$ value \\
\hline Age & & $59 \pm 15$ & $75 \pm 6$ & $<0.001$ \\
\hline Sex, male & & $32(74)$ & $10(67)$ & 0.03 \\
\hline Left dominant coronary circulation & & $7(16)$ & $0(0)$ & 0.05 \\
\hline \multirow[t]{3}{*}{ Specific CAA } & RCA from left cusp & $14(33)$ & $4(27)$ & 0.15 \\
\hline & LCx from right cusp & $21(49)$ & $8(53)$ & 0.14 \\
\hline & LMCA or LAD from right cusp & $8(19)$ & $3(20)$ & 0.54 \\
\hline \multirow[t]{2}{*}{ Aorta measurements } & Sinus of Valsalva, $\mathrm{mm}$ & $36 \pm 5$ & $32 \pm 5$ & 0.01 \\
\hline & Mid-ascending aorta, mm & $39 \pm 7$ & $35 \pm 3$ & 0.04 \\
\hline \multirow{5}{*}{$\begin{array}{l}\text { Preoperative indication for valve } \\
\text { surgery }\end{array}$} & Aortic stenosis & $27(62)$ & $11(73)$ & 0.14 \\
\hline & Aortic regurgitation & $5(12)$ & $0(0)$ & 0.08 \\
\hline & Aortic stenosis/regurgitation & $9(21)$ & $4(27)$ & 0.57 \\
\hline & Aortic regurgitation, total & $14(33)$ & $4(27)$ & 0.15 \\
\hline & Ascending aorta repair/replacement & $14(33)$ & $0(0)$ & $<0.001$ \\
\hline \multirow[t]{6}{*}{ Surgical pathology finding } & Aortic intimal proliferation & $1(2)$ & $0(0)$ & $N / A^{*}$ \\
\hline & Aortic medial degeneration & $4(9)$ & $0(0)$ & 0.56 \\
\hline & Aortic medial necrosis & 1 (2) & $0(0)$ & $N / A^{*}$ \\
\hline & Aortic medial dissection & 1 (2) & $0(0)$ & $N / A^{*}$ \\
\hline & Hypertrophic cardiomyopathy & $0(0)$ & $1(7)$ & $N / A^{*}$ \\
\hline & Amyloidosis, TTR type & $0(0)$ & $1(7)$ & $N / A^{*}$ \\
\hline \multirow{5}{*}{$\begin{array}{l}\text { Surgical identification of any/ } \\
\text { additional congenital heart disease }\end{array}$} & Ventricular septal defect & $1(2)$ & $0(0)$ & $N / A^{*}$ \\
\hline & Ebstein anomaly and secundum atrial septal defect & $1(2)$ & $0(0)$ & $N / A^{*}$ \\
\hline & Double outlet right ventricle & $1(2)$ & $0(0)$ & $N / A^{*}$ \\
\hline & Subaortic membrane & $1(2)$ & $0(0)$ & $N / A^{*}$ \\
\hline & Any/additional congenital heart disease, total & $4(9)$ & $0(0)$ & 0.56 \\
\hline
\end{tabular}

Data expressed as mean \pm SD or as number (percentage).

*Not clinically significant.

ACAOS, anomalous origination of a coronary artery from the opposite sinus; BAV, bicuspid aortic valve; BMI, body mass index; BSA, body surface area; DBP, diastolic blood pressure; LAD, left anterior descending artery; LCx, left circumflex artery; LMCA, left main coronary artery; $\mathrm{N} / \mathrm{A}$, not applicable; RCA, right coronary artery; SBP, systolic blood pressure; TAV, tricuspid aortic valve; TTR, transthyretin. 
Table 4 Predictors for ACAOS in patients with BAV and patients with TAV $(n=4050)$

\begin{tabular}{|c|c|c|c|c|}
\hline Variable & $\begin{array}{l}\text { Univariate analysis } \\
\text { OR }(95 \% \mathrm{Cl})\end{array}$ & P value & $\begin{array}{l}\text { Multivariate analysis } \\
\text { OR }(95 \% \mathrm{CI})\end{array}$ & P value \\
\hline Age & $0.97(0.96$ to 0.99$)$ & 0.001 & 0.99 (0.96 to 1.01$)$ & 0.39 \\
\hline BAV & 2.04 (1.13 to 3.7$)$ & 0.02 & 3.29 (1.26 to 8.6$)$ & 0.02 \\
\hline Mid-ascending aorta & 0.95 (0.89 to 1.0$)$ & 0.08 & $0.93(0.87$ to 0.98$)$ & 0.01 \\
\hline Aortic stenosis & 0.76 (0.45 to 1.31$)$ & 0.33 & & \\
\hline Sinus of Valsalva & $0.96(0.88$ to 1.05$)$ & 0.39 & & \\
\hline Sex, male & 1.26 (0.69 to 2.28$)$ & 0.44 & & \\
\hline Aortic regurgitation & 1.39 (0.55 to 3.52) & 0.48 & & \\
\hline Left dominant coronary circulation & 0.99 (0.47 to 2.09) & 0.97 & & \\
\hline
\end{tabular}

Data expressed as OR (95\% Cl).

ACAOS, anomalous origination of a coronary artery from the opposite sinus; BAV, bicuspid aortic valve; TAV, tricuspid aortic valve.

involvement of ventricular endocardium and epicardialderived endothelial cells, such that the coronary arteries likely form on the surface of the heart and grow up to join the aortic root, a result of complex cell signalling pathways. ${ }^{2}$ It has been postulated that perhaps an abnormally formed aortic valve does not provide the correct molecular landmarks, resulting in abnormal connection of the coronary arteries to the root. ${ }^{11}{ }^{19}$ While the molecular processes are not completely understood, our study demonstrates that the correlation between the two clearly exists; we found in a large number of patients that left dominance is more prevalent in BAV and that BAV is statistically associated to the presence of ACAOS.

\section{Prior studies}

Current knowledge on the association of BAV-ACAOS is conflicting; Michalowska et al studied 193 patients with BAV and 235 patients with TAV and concluded that the prevalence of CAA was similar in BAV and TAV populations, with seven total patients identified with ACAOS distributed similarly between BAV and TAV. ${ }^{12}$ Conversely, Naito et al investigated CAA prevalence in 345 patients with BAV and 754 patients with TAV and concluded that BAV had a higher prevalence of CAA. ${ }^{13}$ However, the definition of CAA used by the investigators also included separate ostia of the LCx and LAD and high or low take-off of a coronary artery. Although neither the specific prevalence nor the specific clinical impact of ACAOS was delineated, anomalous origin of the RCA was significantly more common in BAV, while anomalous origin of the left coronary artery was much less frequent and not statistically different between patients with BAV and patients with $\mathrm{TAV} .{ }^{13}$ In turn, we demonstrate that overall ACAOS is two times more prevalent in BAV $(1.8 \%$ vs $0.9 \%, \mathrm{p}=0.02$, threefold higher odds), includes all variants of ACAOS, and that anomalous origin of the LCx was the most common ACAOS in both BAV and TAV. The prevalence of ACAOS in large studies of the general population has been estimated to range from $0.6 \%$ to $1 \%{ }^{4}{ }^{20}$ Including both patients with BAV and patients with TAV, Naito et al found a $2.4 \%(27 / 1099)$ prevalence of ACAOS and we found a 1.4\% (58/4050) total prevalence, closer to that reported in the general population.

Finally, while Naito et al noted higher rates of postoperative coronary angiography and percutaneous coronary intervention in patients with $\mathrm{CAA},{ }^{13}$ no patient with CAA in our cohort required either; two complications were resolved intraoperatively. Given the relatively small number of patients with CAA available for study, further research is required to clarify the perioperative risk in the ACAOS population.

\section{ACAOS,BAV phenotype and aortopathy}

With greater attention to BAV variants, we now understand that not all BAV behave alike. A BAV with visible raphe by echocardiography is associated with evidence of valve degeneration and future stenosis. ${ }^{21}$ Patients with genetic syndromes and BAV also have varying patterns of cusp fusion: right non-coronary cusp fusion predominates in patients with Down syndrome, whereas rightleft cusp fusion is the most frequent pattern in those with Turner syndrome, DiGeorge syndrome and Shone syndrome. $^{22}$ It follows, therefore, that perhaps coronary anatomy may correlate with BAV type. A recent study investigated coronary artery anatomy in patients with BAV, comparing those patients with and without observed raphe and demonstrated an increased prevalence of left dominant circulation and separate ostia of the LAD and LCx in patients without raphe. ${ }^{11}$ In our study, the great majority of patients had right-left cusp fusion with present raphe, and there was no significant association observed between valve type and CAA pattern. However, given the overall low total number of patients with ACAOS (43/2371), this likely represents a reflection of the higher frequency of right-left fusion in BAV itself, ${ }^{21}{ }^{23}$ which is usually between $70 \%$ and $80 \%$, and it will require a multicentre study with many thousands of patients to determine whether an association between BAV phenotype and ACAOS really exists. Similarly, we chose to evaluate the association between root size/ascending aorta size and ACAOS, as well as the association between BAV stenosis/regurgitation and ACAOS, 
because they represent different clinical histories for the bicuspid patient which could potentially be genetically determined and thus associated with other congenital abnormalities (ie, ACAOS) ${ }^{23}{ }^{24}$ Roughly, the typical clinical types are the older patient with BAV, either male or female, presenting more often with aortic valve sclerosis/ stenosis and ascending aorta dilatation phenotype, and the younger patient with BAV, usually male, presenting with $\mathrm{AR}$ and root dilatation phenotype. ${ }^{23}$

\section{Clinically significant CAA in patients with BAV}

Our data demonstrate that all phenotypes of ACAOS are seen in BAV and that prevalence is twice as common in BAV. Furthermore, BAV was an independent predictor for the presence of ACAOS. The clinical perspective of this statistically significant finding is that approximately 2/100 patients with BAV requiring AVR will have ACAOS vs $1 / 100$ of TAV-AVR. Therefore, the absolute risk for both BAV and TAV populations remains low. Given the potential 'malignant' nature of ACAOS, coronary imaging seems a necessity prior to any cardiac surgery in both groups, but particularly in patients with BAV, to avoid ischaemic events during AVR and root-repair surgeries. However, clinicians may tend to forego a presurgical coronary angiogram in patients with BAV $<50$ years old, and our paper suggests that all patients with BAV should undergo coronary artery anatomy evaluation before surgery. Fortunately, many physicians and surgeons evaluate patients with BAV with cardiac CTA before surgery for AVR and/or aorta repair, and our results support this practice. We demonstrate that AVR can be performed safely without significant complication when coronary anatomy is known ahead of time.

\section{LIMITATIONS}

This is a single-centre retrospective study and subject to the inherent bias associated with that type of study. Separate ostia of the LAD and LCx were not considered a CAA in this study due to the high variability in coding that diagnosis at the time of angiogram. Patients without coronary angiography were excluded from our study, and it is possible that some patients with CAA were not recognised. Nonetheless, assuming that the 895 patients with BAV and 294 patients with TAV without known coronary anatomy had both at least $0.9 \%$ prevalence of ACAOS, the prevalence of ACAOS would still be $1.6 \%$ for BAV and $0.9 \%$ for TAV $(p=0.032$ ). Finally, the echocardiographic information was not complete, especially regarding measurements of the aorta (tables 2 and 3); however, the diagnosis of BAV versus TAV was based on the gold-standard proof of pathology examination of excised aortic valves.

\section{CONCLUSION}

Patients with BAV have threefold higher odds of ACAOS compared with patients with TAV and anomalous LCx from the right sinus is the most common. Most BAVACAOS had right-left cusp fusion and present raphe.
All patterns of ACAOS were represented in patients with $\mathrm{BAV}$, and there was no observable association between BAV anatomy and ACAOS type. Patients with known ACAOS have a low incidence of perioperative coronary injury or ischaemic event.

\section{Twitter Hector Michelena @michelenahector}

Contributors All authors contributed to the design, data collection, analysis, drafting and revision of the manuscript. SV, MF, L-TY, JMI and HM have had full access to all the data in the study, and they take full responsibility for the integrity of the data and the accuracy of the data analysis.

Funding The authors have not declared a specific grant for this research from any funding agency in the public, commercial or not-for-profit sectors.

Competing interests None declared.

Patient consent for publication Not required.

Ethics approval The Institutional Review Board at Mayo Clinic, Rochester, Minnesota, USA (IRB ID 09-0 06 122) approved this study and waived informed consent.

Provenance and peer review Not commissioned; externally peer reviewed.

Data availability statement All data relevant to the study are included in the article. Due to confidentiality issues, the datasets and study materials safe guarded by the Health Science Department of Mayo Clinic cannot be made available to outside parties.

Open access This is an open access article distributed in accordance with the Creative Commons Attribution Non Commercial (CC BY-NC 4.0) license, which permits others to distribute, remix, adapt, build upon this work non-commercially, and license their derivative works on different terms, provided the original work is properly cited, appropriate credit is given, any changes made indicated, and the use is non-commercial. See: http://creativecommons.org/licenses/by-nc/4.0/.

ORCID iD

Hector Michelena http://orcid.org/0000-0002-2341-5247

\section{REFERENCES}

1 Click RL, Holmes DR, Vlietstra RE, et al. Anomalous coronary arteries: location, degree of atherosclerosis and effect on survival--a report from the Coronary Artery Surgery Study. J Am Coll Cardiol 1989;13:531-7.

2 Pérez-Pomares JM, de la Pompa JL, Franco D, et al. Congenital coronary artery anomalies: a bridge from embryology to anatomy and pathophysiology--a position statement of the development, anatomy, and pathology ESC Working Group. Cardiovasc Res 2016;109:204-16.

3 Yamanaka O, Hobbs RE. Coronary artery anomalies in 126,595 patients undergoing coronary arteriography. Cathet Cardiovasc Diagn 1990;21:28-40.

4 Angelini P. Coronary artery anomalies: an entity in search of an identity. Circulation 2007;115:1296-305.

5 Spicer DE, Henderson DJ, Chaudhry B, et al. The anatomy and development of normal and abnormal coronary arteries. Cardiol Young 2015;25:1493-503.

6 Steinberger J, Lucas RV, Edwards JE, et al. Causes of sudden unexpected cardiac death in the first two decades of life. Am J Cardiol 1996;77:992-5.

7 Loukas M, Andall RG, Khan AZ, et al. The clinical anatomy of high take-off coronary arteries. Clin Anat 2016;29:408-19.

8 Warnes CA, Williams RG, Bashore TM, et al. ACC/AHA 2008 guidelines for the management of adults with congenital heart disease: a report of the American College of Cardiology/American heart association Task force on practice guidelines (writing Committee to develop guidelines on the management of adults with congenital heart disease). Circulation 2008;118:e714-833.

9 Scholz DG, Lynch JA, Willerscheidt AB, et al. Coronary arterial dominance associated with congenital bicuspid aortic valve. Arch Pathol Lab Med 1980;104:417-8.

10 Lerer PK, Edwards WD. Coronary arterial anatomy in bicuspid aortic valve. Necropsy study of 100 hearts. Br Heart J 1981;45:142-7.

11 Koenraadt WMC, Tokmaji G, DeRuiter MC, et al. Coronary anatomy as related to bicuspid aortic valve morphology. Heart 2016;102:943-9. 
12 Michałowska IM, Hryniewiecki T, Kwiatek P, et al. Coronary artery variants and anomalies in patients with bicuspid aortic valve. $J$ Thorac Imaging 2016;31:156-62.

13 Naito S, Petersen J, Reichenspurner H, et al. The impact of coronary anomalies on the outcome in aortic valve surgery: comparison of bicuspid aortic valve versus tricuspid aortic valve morphotype. Interact Cardiovasc Thorac Surg 2018;26:617-22.

14 Zoghbi WA, Adams D, Bonow RO, et al. Recommendations for noninvasive evaluation of native valvular regurgitation: a report from the American Society of echocardiography developed in collaboration with the Society for cardiovascular magnetic resonance. J Am Soc Echocardiogr 2017;30:303-71.

15 Alegret JM, Calvo N, Ligero C, et al. Dilated aortic root is related to a global aortic dilating diathesis. J Vasc Surg 2010;52:867-71.

16 Anderson RH, Webb S, Brown NA, et al. Development of the heart: (3) formation of the ventricular outflow tracts, arterial valves, and intrapericardial arterial trunks. Heart 2003;89:1110-8.

17 DeFaria Yeh D. Coronary artery variants and bicuspid aortic valve disease: gaining insight into genetic underpinnings. Heart 2018;104:363-4

18 Fernández B, Durán AC, Fernández-Gallego T, et al. Bicuspid aortic valves with different spatial orientations of the leaflets are distinct etiological entities. J Am Coll Cardiol 2009;54:2312-8.
19 Tian X, Hu T, Zhang H, et al. Subepicardial endothelial cells invade the embryonic ventricle wall to form coronary arteries. Cell Res 2013;23:1075-90.

20 Kimbiris D, Iskandrian AS, Segal BL, et al. Anomalous aortic origin of coronary arteries. Circulation 1978;58:606-15.

21 Michelena HI, Desjardins VA, Avierinos J-F, et al. Natural history of asymptomatic patients with normally functioning or minimally dysfunctional bicuspid aortic valve in the community. Circulation 2008;117:2776-84.

22 Niaz T, Poterucha JT, Olson TM, et al. Characteristic morphologies of the bicuspid aortic valve in patients with genetic syndromes. J Am Soc Echocardiogr 2018;31:194-200.

23 Michelena HI, Prakash SK, Della Corte A, et al. Bicuspid aortic valve: identifying knowledge gaps and rising to the challenge from the International bicuspid aortic valve Consortium (BAVCon). Circulation 2014;129:2691-704.

24 Della Corte A, Bancone C, Quarto C, et al. Predictors of ascending aortic dilatation with bicuspid aortic valve: a wide spectrum of disease expression. Eur J Cardiothorac Surg 2007;31:397-405 discussion 04-5. 